TRANSACTIONS OF THE

AMERICAN MATHEMATICAL SOCIETY

Volume 354, Number 8, Pages 3207-3227

S 0002-9947(02)03031-3

Article electronically published on April 3, 2002

\title{
LJUSTERNIK-SCHNIRELMAN THEORY IN PARTIALLY ORDERED HILBERT SPACES
}

\author{
SHUJIE LI AND ZHI-QIANG WANG
}

\begin{abstract}
We present several variants of Ljusternik-Schnirelman type theorems in partially ordered Hilbert spaces, which assert the locations of the critical points constructed by the minimax method in terms of the order structures. These results are applied to nonlinear Dirichlet boundary value problems to obtain the multiplicity of sign-changing solutions.
\end{abstract}

\section{INTRODUCTION}

The minimax method has been used extensively in constructing critical points for functionals defined in Hilbert and Banach spaces as solutions of nonlinear partial differential equations. In particular, when the problems possess symmetry, one constructs multiple critical points by the minimax method; this is the general Ljusternik-Schnirelman type theory (e.g., [7], [35, [42], 443], [44], [21], [32]). When an order structure is present, one can also use fixed point theory, degree arguments and the variational method to construct solutions of differential equations (see for example [3], [15], 16], [22], 23], 33], [34, and references therein), but most of this work has been for positive solutions. In order to study the structure of nodal solutions (i.e., sign-changing solutions), efforts have been made in recent years to link critical point theory with the order structure of the function space (see [8], [9], 10], [12], [13], [17, 25], 26], 27], [39], and references therein). However, little work has been done for invariant functionals under group actions when one expects to obtain multiplicity of critical points. One of the motives of this paper is, in the setting of Ljusternik-Schnirelman type theory, to establish relations between critical points given by the minimax method and the order structure of the spaces. For example, we shall establish a relation between the critical points given by Clark's Theorem (which is a variant of Ljusternik-Schnirelman theory) and the positive and negative cones in the space. The classical result of Clark is the following.

Theorem A ([21]). Let $E$ be a Hilbert space, and assume that $\Phi \in C^{1}(E, \mathbf{R})$ satisfies the $(P S)$ condition and is even and bounded from below. Assume $\Phi(0)=0$, and there is an n-dimensional sphere $S_{\rho}$ centered at 0 with radius $\rho>0$ such that $\sup _{S_{\rho}} \Phi(x)<0$. Then $\Phi$ has at least $n$ pairs of critical points with negative critical values.

Received by the editors November 1, 2001.

2000 Mathematics Subject Classification. Primary 35J20, 35J25, 58E05.

Key words and phrases. Ljusternik-Schnirelman theory, order structure, minimax method, sign-changing solutions. 
Now, suppose there is a closed convex cone $P_{E} \subset E$. Then one may ask what is the location of these $n$ pairs of critical points in relation with $P_{E}$ and $-P_{E}$. Under suitable conditions, we shall prove that $\Phi$ has at least one pair of critical points in $P_{E}$ and $-P_{E}$, and that $\Phi$ has at least $(n-1)$ pairs of critical points in $E \backslash\left(P_{E} \cup-P_{E}\right)$. Several general results of this type will be given in Section 2 ; see Theorems 2.1, 2.2, 2.3, and 2.4, as well as some remarks on possible generalizations. Another motive of our work in this paper is to continue our study in [39] and clarify the locations of critical points produced by linking type theorems in relation with the order structure. With the order structure built in, the geometric conditions required in many linking type theorems can be weakened. This turns out to be very important and useful, because in many applications the technical verification of a linking structure is tedious and troublesome. This type of results will be given in Section 3. Finally, our theory is not confined to situations of cone structures; we may consider the locations of critical points in relation to some given invariant sets of the flow.

In Section 4 we apply our theory to nonlinear elliptic Dirichlet problems. Many known results in the literature can be restated and sharpened to assert the existence of sign-changing solutions. Our novelty is to establish multiplicity of sign-changing solutions. We only select a number of classical problems to show the applications of our theory, and shall not seek optimal conditions for the results.

\section{ACKNOWLEDGEMENTS}

The authors are grateful to E. N. Dancer for his inspiring comments in a private communication on our earlier work [39], which partially motivated our work, especially the results in Section 3; to Z. L. Liu for some interesting discussions; and to S. B. Liu for pointing out an error in an earlier version of the paper. The first author is supported by the "973" program of the Chinese National Science Foundation and the Foundation of Chinese Academy of Sciences. The second author thanks the Institute of Mathematics, Academia Sinica, for hospitality during his visit in December 2000.

\section{LJUSTERNIK-SCHNIRELMAN THEORY REVISITED}

Let us start with Clark's theorem as stated in Section 1, which gives multiplicity results for solutions when applied to nonlinear elliptic boundary value problems. One question arising naturally is, among these solutions, how many are one sign solutions (positive and negative) and how many are sign-changing ones? In this section we prove an abstract generalization of Clark's Theorem, which gives the location of the critical points with respect to a pair of cones in the space. In order to do this in an abstract way, we need to introduce additional structure in the space and impose some conditions, although these conditions are all satisfied very naturally in applications to elliptic boundary value problems, as we shall show in Section 4.

Let $E$ be a Hilbert space and $X \subset E$ a Banach space densely embedded in $E$. Assume that $E$ has a closed convex cone $P_{E}$ and that $P:=P_{E} \cap X$ has interior points in $X$, i.e., $P=\stackrel{\circ}{P} \cup \partial P$, with $\stackrel{\circ}{P}$ the interior and $\partial P$ the boundary of $P$ in $X$. We shall use $\|\cdot\|$ and $\|\cdot\|_{X}$ to denote the norms in $E$ and $X$ respectively. We also use $d_{E}(\cdot, \cdot)$ and $d_{X}(\cdot, \cdot)$ to denote the distances in $E$ and $X$. 
Let $\Phi \in C^{2}(E, \mathbf{R})$. We use the following notation: for $a, b, c \in \mathbf{R}, K=K(\Phi)=$ $\left\{x \in E \mid \Phi^{\prime}(x)=0\right\}, \Phi^{b}=\{x \in E \mid \Phi(x) \leq b\}, K_{c}=\left\{x \in E \mid \Phi(x)=c, \Phi^{\prime}(x)=0\right\}$, $K([a, b])=\left\{x \in E \mid \Phi(x) \in[a, b], \Phi^{\prime}(x)=0\right\}$.

Let us consider a normalized negative gradient flow $\sigma$ for $\Phi$ which is well defined for $(t, u) \in \mathbf{R} \times E$ :

$$
\left\{\begin{array}{l}
\frac{d}{d t} \sigma(t, u)=-\frac{\nabla \Phi(\sigma(t, u))}{1+\|\nabla \Phi(\sigma(t, u))\|}, \\
\sigma(t, u)=u .
\end{array}\right.
$$

The first assumption we make is

$(\Phi) K(\Phi) \subset X$ and $\nabla \Phi: X \rightarrow X$ is $C^{1}$.

Under $(\Phi)$, we have $\sigma(t, x) \in X$ for $x \in X$, and $\sigma$ is continuous in $(t, x) \in \mathbf{R} \times X$.

Definition 2.1. With the flow $\sigma$, we call a subset $A \subset E$ an invariant set if $\sigma(t, A) \subset A$ for $t \geq 0$.

Definition 2.2. Let $W \subset X$ be an invariant set under $\sigma$. We say $W$ is an admissible invariant set for $\Phi$ if (a) $W$ is the closure of an open set in $X$, i.e., $W=\stackrel{\circ}{W} \cup \partial W$; (b) if $u_{n}=\sigma\left(t_{n}, v\right)$ for some $v \notin W$ and $u_{n} \rightarrow u$ in $E$ as $t_{n} \rightarrow \infty$, for some $u \in K$, then $u_{n} \rightarrow u$ in $X$; (c) if $u_{n} \in K \cap W$ is such that $u_{n} \rightarrow u$ in $E$, then $u_{n} \rightarrow u$ in $X$; (d) for any $u \in \partial W \backslash K, \sigma(t, u) \in \stackrel{\circ}{W}$ for $t>0$.

Theorem 2.1. Let $\Phi \in C^{2}(E, \mathbf{R})$, and let $(\Phi)$ hold. Assume $\Phi$ is even, bounded from below, $\Phi(0)=0$, and satisfies the $(P S)$ condition. Assume that the positive cone $P$ is an admissible invariant set for $\Phi$ and $K_{c} \cap \partial P=\emptyset$ for all $c<0$. Suppose there is a linear subspace $F \subset X$ with $\operatorname{dim} F=n$ such that $\sup _{F \cup \partial B_{\rho}} \Phi(x)<0$ for some $\rho>0$, where $B_{\rho}=\{x \in E \mid\|x\| \leq \rho\}$. Then $\Phi$ has at least $n$ pairs of critical points with negative critical values. More precisely:

(i) If $\inf _{P} \Phi \leq \inf _{E} \Phi$, then $\Phi$ has at least one pair of critical points in $\stackrel{\circ}{P} \cup(-\stackrel{\circ}{P})$ and at least $n-1$ pairs of critical points in $X \backslash(P \cup-P)$.

(ii) If $\inf _{E} \Phi<\inf _{P} \Phi$, then $\Phi$ has at least one pair of critical points in $\stackrel{\circ}{P} \cup(-\stackrel{\circ}{P})$ and at least $n$ pairs of critical points in $X \backslash(P \cup-P)$.

In order to prove this theorem we need the notion of genus (e.g., [43], 44]). Let

$$
\Sigma_{X}=\{A \subset X \mid A \text { is closed in } X, A=-A\},
$$

and let $i_{X}(A)$ denote the genus of $A$, which is defined as the least integer $n$ such that there exists an odd continuous map $\varphi:=A \rightarrow S^{n-1}$. Here the continuity is with respect to the topology in $X$. With the topology in $E$, we may also define the genus of $A \in \Sigma_{E}=\{A \subset E \mid A$ is closed in $E, A=-A\}$. For preciseness, we use $i_{X}(\cdot)$ and $i_{E}(\cdot)$ when we use different norms. We refer to 43 and 44 for the following properties of genus.

Proposition 2.1. Let $A, B \in \Sigma_{X}$, and let $h \in C(X, X)$ be an odd homeomorphism. Then:

(i) $A \subset B \Rightarrow i_{X}(A) \leq i_{X}(B)$;

(ii) $i_{X}(A \cup B) \leq i_{X}(A)+i_{X}(B)$;

(iii) $i_{X}(A) \leq i_{X}(h(A))$;

(iv) if $A$ is compact, there exists a neighborhood $N \in \Sigma_{X}$ such that $A \subset \stackrel{\circ}{N} \subset N$ and $i_{X}(N)=i_{X}(A)$;

(v) if $F$ is a linear subspace in $X$ with $\operatorname{dim} F=n$, and if $A \subset F$ is bounded, open and symmetric, then $i_{X}(\partial A)=n$. 
This lemma is still true when we replace $\Sigma_{X}$ by $\Sigma_{E}$, with obvious modifications.

We need some preliminaries. We shall use the notation

$$
S=X \backslash(P \cup-P) .
$$

Lemma 2.1. If $A \in \Sigma_{X}$ with $2 \leq i_{X}(A)<\infty$, then $A \cap S \neq \emptyset$.

Proof. If $A \cap S=\emptyset, A \subset(P \cup-P) \backslash\{0\}$. Then $A=A_{+} \cup A_{-}$with $A_{+} \subset P \backslash\{0\}$, $A_{-} \subset-P \backslash\{0\}$. We easily see that $i_{X}(A)=1$, a contradiction.

Lemma 2.2. Let $A \in \Sigma_{E}$. Then $A \cap X \in \Sigma_{X}$ and $i_{E}(A) \geq i_{X}(A \cap X)$.

Proof. Let $x_{n} \in A \cap X$ be such that $x_{n} \rightarrow x \in X$ in $X$. Then, due to the embedding $e: X \rightarrow E, x_{n} \rightarrow x$ in $E$. Then $x \in A$, and $x \in A \cap X$. So $A \cap X$ is closed in $X$. Let $i_{E}(A)=n$. Then there exists $\varphi: A \rightarrow S^{n-1}$, odd and continuous (in $E$ ). Then $\widetilde{\varphi}=\varphi \circ e: A \cap X \rightarrow S^{n-1}$ is odd and continuous in $X$. Hence $i_{X}(A \cap X) \leq n$.

Lemma 2.3. Let $\Psi: E \rightarrow \mathbf{R}$ be a locally Lipschitz continuous function. Then $\left.\Psi\right|_{X}: X \rightarrow \mathbf{R}$ is also locally Lipschitz continuous in the topology of $X$.

Proof. Let $c>0$ be the embedding constant from $X$ to $E$, i.e., $\|u\| \leq c\|u\|_{X}$, for any $u \in X$. For $u \in X$ fixed, first there exist $\delta>0$ and $L>0$ such that for all $v \in E$ with $\|v-u\|<\delta$ we have $|\Psi(v)-\Psi(u)| \leq L\|v-u\|$. Now, for all $v \in X$ with $\|v-u\|_{X}<\frac{\delta}{c+1}$, we have $|\Psi(v)-\Psi(u)| \leq L\|v-u\| \leq L c\|v-u\|_{X}$.

Next, we state a deformation lemma which will be used repeatedly.

Lemma 2.4. Assume $\Phi \in C^{2}(E, \mathbf{R})$ satisfies $(\Phi)$ and the $(P S)$ condition. Let $W \subset X$ be an admissible invariant set for $\Phi$. Assume $K_{c} \cap \partial W=\emptyset$ for some $c$, so that there is $\delta>0$ such that, writing $K_{c}^{1}=K_{c} \cap \stackrel{\circ}{W}$ and $K_{c}^{2}=K_{c} \cap(X \backslash W)$,

$$
\left(K_{c}^{1}\right)_{4 \delta} \cap\left(K_{c}^{2}\right)_{4 \delta}=\emptyset,
$$

where $\left(K_{c}^{i}\right)_{4 \delta}=\left\{u \in E \mid d_{E}\left(u, K_{c}^{i}\right)<4 \delta\right\}$ for $i=1,2$. Then there is $\varepsilon_{0}>0$ such that for any $0<\varepsilon \leq \varepsilon_{0}$ and any compact subset $A \subset\left(\Phi^{c+\varepsilon} \cap X\right) \cup W$, there exists $\eta \in C([0,1] \times X, X)$ such that

(i) $\eta(t, u)=u$ for $t=0$ or $u \notin \Phi^{-1}([c-3 \varepsilon, c+3 \varepsilon]) \backslash\left(K_{c}^{2}\right)_{\delta}$;

(ii) $\eta\left(1, A \backslash\left(K_{c}^{2}\right)_{3 \delta}\right) \subset \Phi^{c-\varepsilon} \cup W$, and $\eta(1, A) \subset \Phi^{c-\varepsilon} \cup W$ if $K_{c}^{2}=\emptyset$;

(iii) $\eta(t, \cdot)$ is a homeomorphism of $X$ for $t \in[0,1]$;

(iv) $\Phi(\eta(\cdot, u))$ is non-increasing for any $u \in X$;

(v) $\eta(t, W) \subset W$ for any $t \in[0,1]$; and

(vi) if $\Phi$ is even, $\eta$ is odd in $u$.

Proof. First, due to the (PS) condition and (c) of Definition 2.2, we can choose $\varepsilon_{0}>0$ to satisfy the following conditions:

$$
K\left(\left[c-3 \varepsilon_{0}, c+3 \varepsilon_{0}\right]\right) \cap \partial W=\emptyset, \quad K\left(\left[c-3 \varepsilon_{0}, c+3 \varepsilon_{0}\right]\right) \subset\left(K_{c}\right)_{\delta},
$$

and

$$
\frac{\|\nabla \Phi(u)\|^{2}}{1+\|\nabla \Phi(u)\|} \geq \frac{3 \epsilon_{0}}{\delta}, \quad \forall u \in \Phi^{-1}\left(\left[c-3 \varepsilon_{0}, c+3 \varepsilon_{0}\right]\right) \backslash\left(K_{c}\right)_{\delta}
$$

Let

$$
\begin{gathered}
A=\Phi^{-1}\left(\left[c-3 \varepsilon_{0}, c+3 \varepsilon_{0}\right]\right) \backslash\left(K_{c}^{2}\right)_{\delta} \\
B=\Phi^{-1}([c-2 \varepsilon, c+2 \varepsilon]) \backslash\left(K_{c}^{2}\right)_{2 \delta}
\end{gathered}
$$


and for $u \in E$

$$
\psi(u)=\frac{d_{E}(u, E \backslash A)}{d_{E}(u, E \backslash A)+d_{E}(u, B)} .
$$

Then $\psi(u)$ is locally Lipschitz on $E$, and by Lemma 2.3 also on $X$. Then

$$
f(u)=-\psi(u) \frac{\nabla \Phi(u)}{1+\|\nabla \Phi(u)\|}
$$

defines a locally Lipschitz vector field both on $E$ and on $X$. Consider

$$
\left\{\begin{array}{l}
\frac{d}{d t} \sigma(t, x)=f(\sigma) \\
\sigma(0, x)=x \in X
\end{array}\right.
$$

Since $\|f(x)\| \leq 1$ for all $x \in X$, the Cauchy problem has a unique solution $\sigma(t, x)$ continuous on $\mathbf{R} \times X$. Now for fixed $0<\varepsilon \leq \varepsilon_{0}$ and a compact subset $A \subset$ $\left(\Phi^{c+\varepsilon} \cap X\right) \cup W$, we claim that there is $T>0$ such that $\eta(t, x)=\sigma(t T, x)$ satisfies all the properties of Lemma 2.4. We construct $\eta$ in several steps as follows.

Step 1. For any $x \in\left(A \backslash\left(K_{c}^{2}\right)_{3 \delta}\right) \cap \Phi^{-1}([c-2 \varepsilon, c+2 \varepsilon]) \cap W$, by (2.2) and the fact that $W$ is an admissible invariant set we get $\sigma(t, x) \in \stackrel{\circ}{W}$ for $t>0$. Thus by the continuity of the flow in $X$ there are $T_{x}>0$ and $U_{x}$, a neighborhood of $x$ in $X$, such that $\sigma\left(T_{x}, y\right) \in \stackrel{\circ}{W}$ for all $y \in U_{x}$. Therefore, for all $t \geq T_{x}$ and all $y \in U_{x}$, $\sigma(t, y) \in W$.

Step 2. Consider $x \in\left(A \backslash\left(K_{c}\right)_{3 \delta}\right) \backslash W$ satisfying $\Phi(x) \geq c-2 \varepsilon$. Then $\Phi(x) \leq c+\varepsilon$. If $\sigma(t, x)$ remains in $\Phi^{-1}([c-2 \varepsilon, c+2 \epsilon])$ for $t \in[0, \delta]$, we have

$$
\|\sigma(\delta, u)-u\| \leq \int_{0}^{\delta}\|\dot{\sigma}(\tau, u)\| d \tau \leq \int_{0}^{\delta} d \tau \leq \delta
$$

Thus for all $t \in[0, \delta]$ we have $\sigma(t, x) \notin\left(K_{c}\right)_{2 \delta}$ and $\psi(\sigma(t, x))=1$. Then by $(2.3)$

$$
\begin{aligned}
\Phi(\sigma(\delta, x)) & =\Phi(x)+\int_{0}^{\delta} \frac{d}{d \tau} \Phi(\sigma(\tau, x)) d \tau \\
& =c+\varepsilon-\int_{0}^{\delta} \frac{\|\nabla \Phi(\sigma(\tau, x))\|^{2}}{1+\|\nabla \Phi(\sigma(\tau, x))\|} d \tau \\
& \leq c+\varepsilon-3 \varepsilon \\
& =c-2 \varepsilon .
\end{aligned}
$$

By continuity of the flow in $X$, there is a neighborhood $U_{x}$ of $x$ such that for all $y \in U_{x}$ and all $t \geq T_{x}:=\delta$ we have $\Phi(\sigma(t, y)) \leq c-\varepsilon$.

Step 3. Consider $x \in A \cap\left(\left(K_{c}^{1}\right)_{3 \delta} \backslash W\right)$ with $x \in \Phi^{-1}([c-2 \varepsilon, c+2 \varepsilon])$. There are two cases:

(a) there are $t_{n} \rightarrow \infty$ such that $d_{E}\left(\sigma\left(t_{n}, x\right), K([c-2 \varepsilon, c+2 \varepsilon])\right) \rightarrow 0$;

(b) $\inf _{t \geq 0} d_{E}(\sigma(t, x), K([c-2 \varepsilon, c+2 \varepsilon]))=\delta^{\prime}>0$.

In case (a), if there are $z_{n} \in K([c-2 \varepsilon, c+2 \varepsilon])$ and $z_{n} \notin W$ such that

$$
d_{E}\left(\sigma\left(t_{n}, x\right), z_{n}\right) \rightarrow 0
$$

and if $\sigma(t, x)$ remains in $\Phi^{-1}([c-2 \varepsilon, c+2 \varepsilon])$, then by $(2.2)$ it has to travel for at least $\delta$ units of time outside $\left(K_{c}\right)_{2 \delta}$ to go from $\left(K_{c}^{1}\right)_{3 \delta}$ to $\left(K_{c}^{2}\right)_{2 \delta}$. Then the same argument as in Step 2 gives the existence of $T_{x}$ and $U_{x}$. If there are $z_{n} \in K \cap W$ such that $d_{E}\left(\sigma\left(t_{n}, x\right), z_{n}\right) \rightarrow 0$, by the fact that $W$ is an admissible invariant set and that 
$K([c-2 \varepsilon, c+2 \varepsilon])$ is compact, we see that, along a subsequence, $\sigma\left(t_{n}, x\right)$ converges in $X$ to a point $z \in K([c-2 \varepsilon, c+2 \varepsilon])$. Since $z \in \stackrel{\circ}{W}$, for $n$ large, $\sigma\left(t_{n}, x\right) \in \stackrel{\circ}{W}$. Then by the continuity of the flow in $X$, there are $T_{x}>0$ and $U_{x}$, a neighborhood of $x$ in $X$, such that for all $y \in U_{x}$ and all $t \geq T_{x}$ we have $\sigma(t, y) \in W$.

Finally, for case (b), if $\sigma(t, x)$ reaches $\left(K_{c}^{2}\right)_{2 \delta}$ before entering $\Phi^{c-2 \varepsilon}$, then the same argument as in Step 2 gives $T_{x}>0$ and $U_{x}$ such that $\sigma(t, y) \in \Phi^{c-2 \varepsilon}$ for $t \geq T_{x}$ and $y \in U_{x}$. Assume that $\sigma(t, x)$ stays outside of $\left(K_{c}^{2}\right)_{2 \delta}$. First, by the (PS) condition there is $\varepsilon^{\prime}>0$ such that

$$
\frac{\|\nabla \Phi(y)\|^{2}}{1+\|\nabla \Phi(y)\|} \geq \varepsilon^{\prime}, \quad \forall y \in \Phi^{-1}([c-2 \varepsilon, c+2 \varepsilon]) \backslash(K([c-2 \varepsilon, c+2 \varepsilon]))_{\delta^{\prime}} .
$$

Then if $\sigma(t, x)$ remains in $\Phi^{-1}([c-2 \varepsilon, c+2 \varepsilon])$ for $t \in\left[0, \frac{3 \varepsilon}{\varepsilon^{\prime}}\right]$, we have $\psi(\sigma(t, x))=1$ and

$$
\Phi\left(\sigma\left(\frac{3 \varepsilon}{\varepsilon^{\prime}}, x\right)\right)=\Phi(x)+\int_{0}^{\frac{3 \varepsilon}{\varepsilon^{\prime}}} \frac{d}{d \tau} \Phi(\sigma(\tau, x)) d \tau \leq c+\varepsilon-\frac{3 \varepsilon}{\varepsilon^{\prime}} \varepsilon^{\prime}=c-2 \varepsilon .
$$

Again, there are $T_{x}>0$ and $U_{x}$, a neighborhood of $x$ in $X$, such that for all $t \geq T_{x}$ and $y \in U_{x}$ we have $\Phi\left(\sigma\left(T_{x}, y\right)\right) \leq c-\varepsilon$.

Note that $\left(K_{c}^{2}\right)_{3 \delta} \cap X$ is open in $X$. Then $\left(A \backslash\left(K_{c}^{2}\right)_{3 \delta}\right) \cap \Phi^{-1}([c-2 \varepsilon, c+2 \varepsilon])$ is compact in $X$, and can be covered by finitely many $U_{x_{i}}$, say, for $i=1, \ldots, k$. Define $T=\max \left\{T_{x_{1}}, \ldots, T_{x_{k}}\right\}$ and, for $(t, x) \in \mathbf{R} \times X$,

$$
\eta(t, x)=\sigma(t T, x) .
$$

We claim $\eta$ satisfies all the properties of Lemma 2.4. (i), (iii), (iv), (v) and (vi) are obvious. From the above steps, we have $\eta\left(1, A \cap \Phi^{-1}([c-2 \varepsilon, c+2 \varepsilon]) \backslash\left(K_{c}^{2}\right)_{3 \delta}\right) \subset$ $\Phi^{c-\varepsilon} \cup W$. For $x \in A \backslash\left(K_{c}^{2}\right)_{3 \delta}$ with $\Phi(x)>c+2 \varepsilon$, we have $x \in W$; thus $\eta(1, x) \in W$. For $x \in A \backslash\left(K_{c}^{2}\right)_{3 \delta}$ with $\Phi(x)<c-2 \varepsilon$, by (iv), $\eta(1, x) \in \Phi^{c-2 \varepsilon}$. Thus (ii) is proved.

Remark 2.1. (1) It is easy to check that the union and intersection of a finite number of admissible invariant sets for $\Phi$ is still an admissible invariant set for $\Phi$.

(2) From the proof, we see $\varepsilon_{0}$ is given by (2.2) and (2.3).

Proof of Theorem 2.1. We prove (i), and mention necessary changes for (ii). Since $\inf _{P}(\Phi) \leq \inf _{E}(\Phi)<0$, we may perform the usual minimization argument. Let $u_{n} \in P$ be such that $\Phi\left(u_{n}\right) \rightarrow \inf _{P} \Phi$. Using the flow $\sigma$ if necessary, we may assume $\Phi^{\prime}\left(u_{n}\right) \rightarrow 0$. By the (PS) condition, $u_{n}$ converges to a critical point $u$ in $E$. But $P$ is closed in $E$ and $K_{c} \cap \partial P=\emptyset$ for $c<0$, and so we get $u \in \stackrel{\circ}{P}$, which gives a pair of critical points in $\stackrel{\circ}{P} \cup-\stackrel{\circ}{P}$.

Now for $m \geq 1$ define

$$
\Sigma_{m}=\left\{A \subset \Sigma_{X} \mid A \text { is compact, } i_{X}(A) \geq m\right\},
$$

and for $m \geq 2$ define

$$
c_{m}=\inf _{A \in \Sigma_{m}} \sup _{A \cap S} \Phi(x),
$$

where $S=X \backslash(P \cup(-P))$. By Lemma 2.1,

$$
-\infty<c_{2} \leq \cdots \leq c_{n}<0 .
$$

We claim that, for $m=2, \ldots, n$,

$$
K_{c_{m}} \cap S \neq \emptyset,
$$


and $K_{c_{m}} \cap S$ is closed in $X$ and $E$. We also claim that if, for some $m \geq 1$ and $k \geq 2$ with $m+k \leq n$,

$$
c:=c_{m+1}=c_{m+2}=\cdots=c_{m+k}
$$

then

$$
i_{E}\left(K_{c} \cap S\right) \geq k
$$

To prove (2.4), we assume $K_{c_{m}} \cap S=\emptyset$ for contradiction. Take $W=P \cup-P$ in Lemma 2.4. Then $K_{c_{m}} \cap(X \backslash \stackrel{\circ}{W})=\emptyset$, i.e., $K_{c_{m}}^{2}=\emptyset$. Then there is $\varepsilon_{0}>0$ such that when we take $A \in \Sigma_{m}$ such that $\sup _{A \cap S} \Phi(x) \leq c+\varepsilon$, with $\varepsilon \leq \varepsilon_{0}$, there exists $\eta \in C([0,1] \times X, X)$ satisfying (i) - (vi) of Lemma 2.4 . Take $\tilde{A}=\bar{\eta}(1, A)$. Then by (iii) of Proposition 2.1, $\tilde{A} \in \Sigma_{m}$. By (ii) of Lemma 2.4,

$$
\tilde{A} \subset \Phi^{c-\varepsilon} \cup W \text {. }
$$

Then

$$
\sup _{\tilde{A} \cap S} \Phi(x) \leq c-\varepsilon,
$$

a contradiction.

To prove (2.5), we use Lemma 2.4 again. By the proof of Lemma $2.4, K_{c}^{1}=$ $K_{c} \cap \stackrel{\circ}{W}$ and $K_{c}^{2}=K_{c} \cap S$ are both compact in $E$. By Proposition 2.1, there is a closed neighborhood $N$ in $E$ with $K_{c}^{2} \subset \stackrel{\circ}{N} \subset N$ such that $i_{E}(N)=i_{E}\left(K_{c}^{2}\right)$. Without loss of generality, we may assume $N=\overline{\left(K_{c}^{2}\right)_{3 \delta}}$ in the $E$-norm, with $\delta$ satisfying $\left(K_{c}^{2}\right)_{4 \delta} \cap\left(K_{c}^{1}\right)_{4 \delta}=\emptyset$ as in Lemma 2.4. By Lemma 2.2,

$$
i_{X}(N \cap X) \leq i_{E}(N)=i_{E}\left(K_{c}^{2}\right) .
$$

For the $\delta>0$ given, by Lemma 2.4 there exists $\varepsilon_{0}>0$ such that when we take $A \in$ $\Sigma_{m+k}$ satisfying $\sup _{A \cap S} \Phi(x) \leq c+\varepsilon$ with $\varepsilon \leq \varepsilon_{0}$, there exists $\eta \in C([0,1] \times X, X)$ satisfying (i)-(vi) of Lemma 2.4. Then, by (ii) of Lemma 2.4,

$$
\eta\left(1, A \backslash\left(K_{c}^{2}\right)_{3 \delta}\right) \subset \Phi^{c-\varepsilon} \cup W .
$$

Then we have

$$
i_{X}\left(\eta\left(1, A \backslash\left(K_{c}^{2}\right)_{3 \delta}\right)\right) \leq m,
$$

for otherwise $i_{X}\left(\eta\left(1, A \backslash\left(K_{c}^{2}\right)_{3 \delta}\right)\right) \geq m+1$ and

$$
c=c_{m+1} \leq \sup _{\eta\left(1, A \backslash\left(K_{c}^{2}\right)_{3 \delta}\right) \cap S} \Phi(x) \leq \sup _{\left(\Phi^{c-\varepsilon} \cup W\right) \cap S} \Phi(x) \leq \sup _{\Phi^{c-\varepsilon}} \Phi(x) \leq c-\varepsilon,
$$

a contradiction. Now by (i),(ii) and (iii) of Proposition 2.1, and (iii) of Lemma 2.4, we have

$$
\begin{aligned}
m+k & \leq i_{X}(A) \leq i_{X}(A \backslash(\stackrel{\circ}{N} \cap X))+i_{X}(N \cap X) \\
& \leq i_{X}\left(\eta\left(1, A \backslash\left(K_{c}^{2}\right)_{3 \delta}\right)+i_{E}(N) \leq m+i_{E}\left(K_{c}^{2}\right),\right.
\end{aligned}
$$

which gives $i_{E}\left(K_{c} \cap S\right) \geq k$.

For case (ii), where $\inf _{E} \Phi(x)<\inf _{P} \Phi(x)$, we may also define

$$
c_{1}=\inf _{A \in \Sigma_{1}} \sup _{A \cap S} \Phi(x) .
$$

Then $\inf _{E} \Phi(x)<\inf _{P} \Phi(x)$ implies $c_{1}<\inf _{P} \Phi(x)$ and $c_{1}<0$. If we take $\varepsilon>0$ so that $c_{1}+\varepsilon<\min \left\{\inf _{P} \Phi(x), 0\right\}$, we can follow the same argument as above to 
show $c_{1}$ is a critical value and $K_{c_{1}} \cap S \neq \emptyset$. Also, if $c_{1}=c_{2}=\cdots=c_{k}$, then we can show similarly that $i_{E}\left(K_{c_{1}} \cap S\right) \geq k$. We omit the details here.

Our argument can be used to obtain the following more general version of Clark's Theorem. We omit the proof here.

Theorem 2.2. Let $\Phi \in C^{2}(E, \mathbf{R})$, and let $(\Phi)$ hold. Assume $\Phi$ is even, $\Phi(0)=0$, and $\Phi$ satisfies the $(P S)$ condition. Assume that $P$ is an admissible invariant set for $\Phi$, and $K_{c} \cap \partial P=\emptyset$ for all $c<0$. Suppose there exist linear subspaces $F \subset X$ and $H \subset E$ with $\operatorname{dim} F=n, \operatorname{codim} H=k \geq 1$, such that for some $\rho>0$, $\sup _{F \cap \partial B_{\rho}(0)} \Phi(x)<0$, and let $\inf _{H} \Phi(x)>-\infty$. Then $\Phi$ has at least $(n-k)$ pairs of critical points in $X \backslash(P \cup-P)$ with negative critical values.

A dual version of this is the following.

Theorem 2.3. Let $\Phi \in C^{2}(E, \mathbf{R})$ and let $(\Phi)$ hold. Assume $\Phi$ is even, $\Phi(0)=0$, and $\Phi$ satisfies the $(P S)$ condition. Assume that $P$ is an admissible invariant set for $\Phi$, and $K_{c} \cap \partial P=\emptyset$ for all $c>0$. Suppose there exist linear subspaces $F \subset X$ and $H \subset E$ with $\operatorname{dim} F=n$, codim $H=k$, such that for some $\rho>r>0$, $\inf _{H \cap \partial B_{r}(0)} \Phi(x)>0$ and $\sup _{F \cap \partial B_{\rho}(0)} \Phi(x) \leq 0$. Then, if $k \geq 1$ ( $k=0$ resp. $), \Phi$ has at least $(n-k)((n-1)$ resp. $)$ pairs of critical points in $X \backslash(P \cup-P)$ with positive critical values.

Let us give another result, which is closer to the spirit of Ljusternik-Schnirelman theory, aiming at applications to nonlinear eigenvalue problems. Let $B_{r}=\{u \in$ $E \mid\|u\|=r\}$ with $r>0$.

Theorem 2.4. Let $\Phi \in C^{2}(E, \mathbf{R})$. Let $\Phi$ be even. Suppose $r>0,\left.\Phi\right|_{\partial B_{r}}$ satisfies $(P S), K\left(\left.\Phi\right|_{\partial B_{r}}\right) \subset X \cap \partial B_{r}$ and $\left.\Phi\right|_{\partial B_{r}}$ is bounded from below. Let $P \cap \partial B_{r} \subset X$ be an admissible invariant set for $\left.\Phi\right|_{\partial B_{r}}$. Assume $K\left(\left.\Phi\right|_{\partial B_{r}}\right) \cap \partial P=\emptyset$. Then $\left.\Phi\right|_{\partial B_{r}}$ has infinitely many distinct pairs of critical points in $\left(\partial B_{r} \cap X\right) \backslash(P \cup-P)$.

Proof. We sketch the proof here. That $\left.\Phi\right|_{\partial B_{r}}$ possesses infinitely many pairs of critical points follows from, e.g. to [44]. The question is whether they belong to $P$ and $-P$. We need a procedure similar to the one we used earlier to rule this out.

Let $\widetilde{\Sigma}_{X}=\left\{A \subset X \cap \partial B_{r} \mid A\right.$ compact, $\left.A=-A\right\}$ and

$$
\begin{gathered}
\widetilde{\Sigma}_{m}=\left\{A \in \widetilde{\Sigma}_{X} \mid i_{X}(A) \geq m\right\}, \\
c_{m}=\inf _{A \in \widetilde{\Sigma}_{m}} \sup _{A \cap S} \Phi(x), \text { with } S=X \backslash(P \cup-P) .
\end{gathered}
$$

Then we may follow the idea of the proof of Theorem 2.1 to show that for $m \geq 2$,

$$
K_{c_{m}} \cap\left(\partial B_{r} \backslash(P \cup-P)\right) \neq \emptyset
$$

and if $c_{m}=c_{m+1}=\cdots=c_{m+k}$ for some $m \geq 2, k \geq 2$, then there are infinitely many pairs of critical points in $K_{c_{m}} \cap\left(\partial B_{r} \backslash(P \cup-P)\right)$. We leave the details to interested readers.

Remark 2.2. Obviously, our theory can be set up for $C^{2-0}$ functionals without any changes, since $\nabla \Phi(u)$ is locally Lipschitz continuous. For $C^{1}$ functionals, one may try to approximate them by $C^{2}$ functionals. We shall give an example for which the variational functional cannot be $C^{2}$, and an approximation procedure has to be used. (See Theorem 4.6.) 
Remark 2.3. Obviously, our results can be generalized to $G$-invariant functionals for more general $G$-group actions, for example, $G=S^{1}, Z_{p}$. Instead of the notion of genus, one can use the $S^{1}$-index theory, $Z_{p}$-index theory, etc. (e.g., [30], [46]). We leave the statements of the results to the readers.

Remark 2.4. In all the theorems above, the positive and negative cones $P \cup(-P)$ can be changed to a symmetric admissible invariant set $W$ such that for any $A$ with $i_{X}(A) \in[2, \infty)$ we have $A \cap(X \backslash W) \neq \emptyset$. Then all theorems $2.1-2.4$ are still valid.

Remark 2.5. In all the theorems above, we have used the negative gradient flow. Checking the proofs, one can replace this flow by any flow with similar properties in Lemma 2.4.

Remark 2.6. Another result of constructing critical points for even functionals will be given at the end of Section 3, which is related to linking type theorems.

\section{LINKING THEOREMS IN PARTIALLY ORDERED SPACES}

Generalizing the Mountain Pass Theorem, many people have used minimax theory to prove linking type theorems (e.g., 11], [17, 44, and references therein). In [39], we have proved a Mountain Pass Theorem with the order structure built in, giving the location of the mountain pass critical point with respect to the structure of some invariant subsets under the flow. Following the idea of [39], we continue our study in this direction, namely, proving minimax theorems in the presence of some invariant subsets and giving the location of the critical point produced in relation to the invariant subsets. We shall prove a linking theorem in a very general context which gives the location of the critical point produced by the linking structure with respect to some invariant subsets.

Let us recall that $(Q, T)$ is a linking (here $Q \subset E$ is a closed subset with boundary $\partial Q$ and $T \subset E$ is closed) if (i) $\partial Q \cap T=\emptyset$, and (ii) for any $\gamma \in C(Q, E),\left.\gamma\right|_{\partial Q}=i d$, we have $\gamma(Q) \cap T \neq \emptyset$. (See, e.g., [11, 18], [44] for more general versions.)

A typical linking type theorem is as follows, e.g., [18], 44].

Theorem B. Let $\Phi \in C^{1}(E, \mathbf{R})$ and let $(Q, T)$ be a linking. There exist $\beta>\alpha$ such that

$$
\sup _{Q} \Phi(x)<\infty \text { and } \sup _{\partial Q} \Phi(x) \leq \alpha<\beta \leq \inf _{T} \Phi(x) .
$$

If $(P S)_{c}$ holds for

$$
c=\inf _{\gamma \in \Gamma} \sup _{\gamma(Q)} \Phi(x),
$$

where

$$
\Gamma=\left\{\gamma \in C(Q, E)|\gamma|_{\partial Q}=i d\right\}
$$

then $c$ is a critical value of $\Phi$.

The main result of this section is the following.

Theorem 3.1. Let $\Phi \in C^{2}(E, \mathbf{R})$ satisfy $(\Phi)$. Let $W \subset X$ be an admissible invariant set for $\Phi$. Let $(Q, T)$ be a linking such that $T \subset E \backslash W, Q \subset X$ is compact, and for any $\gamma \in \Gamma=\left\{\gamma \in C(Q, X)|\gamma|_{\partial Q \cap S}=i d, \gamma(\partial Q \cap W) \subset W\right\}$ we have $\gamma(Q) \cap S \neq \emptyset$, where $S=X \backslash W$. Assume that there exists $\beta>\alpha$ such that

$$
\sup _{\partial Q \cap S} \Phi(x) \leq \alpha<\beta \leq \inf _{T} \Phi(x)
$$


and $\sup _{Q} \Phi(x)<\infty$. Let

$$
c=\inf _{\gamma \in \Gamma} \sup _{\gamma(Q) \cap S} \Phi(x) .
$$

Then $c \geq \beta$, and if $K_{c} \cap \partial W=\emptyset$, then $K_{c} \cap S \neq \emptyset$.

Proof. First we show that $\infty>c \geq \beta$. $\sup _{Q} \Phi(x)<\infty$ implies $c<\infty$. So it suffices to show that, for any $\gamma \in \Gamma$,

$$
\gamma(Q) \cap T \cap S \neq \emptyset .
$$

This is true because $\gamma(Q) \cap T \neq \emptyset$ and $\gamma(Q) \subset X$ imply $\gamma(Q) \cap T \cap X \neq \emptyset$. But $T \cap X \subset S$. We get $\gamma(Q) \cap T \cap S \neq \emptyset$. Thus

$$
\sup _{\gamma(Q) \cap S} \Phi(x) \geq \sup _{\gamma(Q) \cap T \cap S} \Phi(x) \geq \inf _{\gamma(Q) \cap T} \Phi(x) \geq \beta .
$$

Next, we show that if $K_{c} \cap \partial W=\emptyset$, then $K_{c} \cap S \neq \emptyset$. If $K_{c} \cap S=\emptyset$, we produce a contradiction as follows. Now, we take $\varepsilon>0$ so that $c-2 \varepsilon>\alpha$, and take $\gamma \in \Gamma$ such that $\sup _{\gamma(Q) \cap S} \leq c+\varepsilon$. By Lemma 2.4, for this $\varepsilon$ and $A=\gamma(Q)$, there exists $\eta \in C([0,1] \times X, X)$ satisfying (i) $-(\mathrm{vi})$, which in particular imply

$$
\eta(1, \gamma(Q)) \subset \Phi^{c-\varepsilon} \cup W .
$$

Consider $\gamma_{1}=\eta(1, \gamma(\cdot))$. Then for $x \in(\partial Q) \cap W$ we have $\gamma_{1}(x)=\eta(1, \gamma(x)) \in W$, since $\gamma(x) \in W$ and $\eta(1, W) \subset W$. For $x \in(\partial Q) \cap S$, since $\Phi(x) \leq \alpha$, we have $\eta(1, \gamma(x))=\eta(1, x)=x$. Hence $\gamma_{1} \in \Gamma$. But

$$
\sup _{\gamma_{1}(Q) \cap S} \Phi(x) \leq \sup _{\left(\Phi^{c-\varepsilon} \cup W\right) \cap S} \Phi \leq \sup _{\Phi^{c-\varepsilon}} \Phi(x) \leq c-\varepsilon,
$$

a contradiction.

Remark 3.1. In case $\partial Q \subset W,(3.1)$ does not make sense. However, in this case, we can still define

$$
c=\inf _{\gamma \in \Gamma} \sup _{\gamma(Q) \cap S} \Phi(x),
$$

where

$$
\Gamma=\{\gamma \in C(Q, X) \mid \gamma(\partial Q) \subset W\} .
$$

By the same proof, we can show if $c$ is finite, then $K_{c} \cap \bar{S} \neq \emptyset$.

Example 3.1. With the positive cone $P$ in $X$ and its interior $\stackrel{\circ}{P}$, we may define a partial order relation: $u, v \in X, u>v \Leftrightarrow u-v \in P \backslash\{0\} ; u \gg v \Leftrightarrow u-v \in \stackrel{\circ}{P}$. An order interval with interior $[u, v]$ for $u \ll v$ is defined as $[u, v]=\{\omega \in X \mid u \leq \omega \leq v\}$. Then we have the following corollary.

Corollary 3.1. Let $\Phi \in C^{2}(E, \mathbf{R})$ satisfy $(\Phi)$ and the $(P S)$ condition. Let $W_{1}, W_{2}$, and $W$ be three admissible invariant sets for $\Phi$ such that $W_{1} \subset W, W_{2} \subset W$, $W_{1} \cap W_{2}=\emptyset$. Define

$$
c_{1}=\inf _{\gamma \in \Gamma} \sup _{\gamma(I) \cap S} \Phi(x),
$$

where

$$
\Gamma=\left\{\gamma \in C(I, W) \mid \gamma(0) \in W_{1}, \gamma(1) \in W_{2}\right\}
$$

and $I=[0,1], S=W \backslash\left(W_{1} \cup W_{2}\right)$. If $c$ is well-defined and $K_{c} \cap\left(\partial\left(W_{1} \cup W_{2}\right)\right)=\emptyset$, then $K_{c} \cap S \neq \emptyset$. Furthermore, if $K_{c} \cap S$ are isolated, there exists $x_{0} \in K_{c} \cap S$ 
such that $x_{0}$ is a mountain-pass point, and $C_{1}\left(\Phi, x_{0}\right) \neq 0$. Here $C_{q}\left(\Phi, x_{0}\right)$ are the critical groups of $\Phi$ at $x_{0}$ (cf. [17]).

Using Remark 3.1, we may deduce this from Theorem 3.1. This corollary is a slightly more general version of the Mountain Pass Theorem in order intervals in [39], where we considered a more concrete situation. The proof in [39] uses a different idea.

Example 3.2. Let us consider another example which has been used in studying nonlinear elliptic problems with superlinear nonlinearity. Let $E=E_{1} \oplus E_{1}^{\perp}$ with $\operatorname{dim} E_{1} \geq 1$. Let $y \in E_{1}^{\perp}$ with $\|y\|=1$, and for $R>0$ define $Q=\left\{e=e_{1}+\right.$ ty $\left.\in E \mid e_{1} \in E_{1},\left\|e_{1}\right\| \leq R, 0 \leq t \leq R,\|e\| \leq R\right\}$. For $0<r<R$, define $T=\left\{e \in E_{1}^{\perp} \mid\|e\|=r\right\}$. Then it is well known that $(Q, T)$ is a linking (e.g., [17], [44).

Corollary 3.2. Let $\Phi \in C^{2}(E, \mathbf{R})$ satisfy $(\Phi)$ and $(P S)$. Assume $W=P \cup(-P)$ is an admissible invariant set for $\Phi$. Assume, as in Example 3.2, that $(Q, T)$ is given satisfying $Q \subset X$ and $P \cup(-P) \subset E \backslash\left(E_{1}^{\perp} \backslash\{0\}\right)$. Assume that, for some $\alpha<\beta$,

$$
\max _{\partial Q \cap S} \Phi \leq \alpha<\beta \leq \inf _{T} \Phi .
$$

If $K_{c} \cap \partial W=\emptyset$, then $K_{c} \cap S \neq \emptyset$, where

$$
c=\inf _{\gamma \in \Gamma} \max _{\gamma(Q) \cap S} \Phi(u),
$$

and

$$
\Gamma=\left\{\gamma \in C(Q, X)|\gamma|_{\partial Q \cap S}=I d, \gamma(\partial Q \cap W) \subset W\right\}
$$

When the functional is even, the conditions can be weakened. In fact, we have the following result.

Corollary 3.3. Let $\Phi \in C^{2}(E, \mathbf{R})$ satisfy $(\Phi)$ and $(P S)$. Assume $\Phi$ is even and $W=P \cup(-P)$ is an admissible invariant set for $\Phi$. Assume, as in Example 3.2, that $(Q, T)$ is given satisfying $Q \subset X$ and $P \cup(-P) \subset E \backslash\left(E_{1}^{\perp} \backslash\{0\}\right)$. Let $Q_{1}=\{u \in Q \mid\|u\|=R\}$. Assume that, for some $\alpha<\beta$,

$$
\max _{Q_{1}} \Phi \leq \alpha<\beta \leq \inf _{T} \Phi .
$$

If $K_{c} \cap \partial W=\emptyset$, then $K_{c} \cap S \neq \emptyset$, where

$$
c=\inf _{\gamma \in \Gamma} \max _{\gamma(Q) \cap S} \Phi(u),
$$

and

$$
\Gamma=\left\{\gamma \in C(Q, X)|\gamma|_{Q_{1}}=I d,\left.\gamma\right|_{B_{R} \cap E_{1}} \text { is odd }\right\} .
$$

Proof. By the argument of [38], for any $\gamma \in \Gamma, \gamma(Q) \cap T \neq \emptyset$. Also, we know $T \cap X \subset S$; so $\gamma(Q) \cap S \neq \emptyset$. Then arguments similar to the above show that $K_{c} \cap S \neq \emptyset$. We leave the details to the readers.

Finally, we give a version of the symmetric Mountain Pass Theorem ([44]).

Theorem 3.2. Assume $\Phi \in C^{2}(E, \mathbf{R})$ is even, $\Phi(0)=0$, and $\Phi$ satisfies $(P S)_{c}$ for $c>0$. Assume that $P$ is an admissible invariant set for $\Phi$, and $K_{c} \cap \partial P=\emptyset$ for all $c>0$. Assume $E=\overline{\bigoplus_{j=1}^{\infty} E_{j}}$, where the $E_{j}$ are finite dimensional subspaces of $X$, and for each $k$, let $Y_{k}=\bigoplus_{j=1}^{k} E_{j}$ and $Z_{k}=\overline{\bigoplus_{j=k}^{\infty} E_{j}}$. Assume that for each $k$ there 
exist $\rho_{k}>r_{k}>0$ such that $\varlimsup_{k \rightarrow \infty} a_{k}<\infty$, where $a_{k}:=\max _{Y_{k} \cap \partial B_{\rho_{k}}(0)} \Phi(x), b_{k}:=$ $\inf _{Z_{k} \cap \partial B_{r_{k}}(0)} \Phi(x) \rightarrow \infty$ as $k \rightarrow \infty$. Then $\Phi(x)$ has a sequence of critical points $u_{n} \in X \backslash(P \cup(-P))$ such that $\Phi\left(u_{n}\right) \rightarrow \infty$ as $n \rightarrow \infty$, provided $Z_{k} \cap \partial B_{\rho_{k}}(0) \cap P=\emptyset$ for large $k$.

Proof. Let

$$
c_{k}=\inf _{\gamma \in \Gamma_{k}} \sup _{u \in \gamma\left(B_{k}\right) \cap S} \Phi(u),
$$

where $B_{k}=\left\{u \in Y_{k} \mid\|u\| \leq \rho_{k}\right\}$ and $\Gamma_{k}=\left\{h \in C\left(B_{k}, X\right) \mid h\right.$ is odd, and $h(u)=$ $u$ if $\left.\|u\|=\rho_{k}\right\}$.

We claim that for any $\gamma \in \Gamma_{k}, \gamma\left(B_{k}\right) \cap S \cap\left(Z_{k} \cap \partial B_{r_{k}}\right) \neq \emptyset$. By an application of the Borsuk-Ulam theorem, one easily sees that $\gamma\left(B_{k}\right) \cap\left(Z_{k} \cap \partial B_{r_{k}}\right) \neq \emptyset$. Since $\left(Z_{k} \cap \partial B_{r_{k}}\right) \cap P=\emptyset$, we have proved the claim. Then this shows that $c_{k} \geq b_{k} \rightarrow \infty$ as $k \rightarrow \infty$.

To show $K_{c_{k}} \cap S \neq \emptyset$, we follow the same argument as in the proof of Theorem 2.1. If $K_{c_{k}} \cap S=\emptyset$, there exists $\varepsilon_{0}>0$ such that $c_{k}-3 \varepsilon>a_{k}$. When we take $0<\varepsilon<\varepsilon_{0}$ and $h \in \Gamma_{k}$ such that $\sup _{h\left(B_{k}\right) \backslash S} \Phi(u) \leq c_{k}+\varepsilon$, by Lemma 2.4 there is a map $\eta(t, u) \in C([0,1] \times X, X)$ such that $\eta\left(1, h\left(B_{k}\right)\right) \subset \Phi^{c-\varepsilon} \cup(P \cup(-P))$. Then $\gamma_{1}(u)=\eta(1, \gamma(u)) \in \Gamma_{k}$, since $\eta$ is odd in $u$ and $\eta(t, u)=u$ for $u \in \Phi^{c_{k}-3 \varepsilon}$. But, $\sup _{h\left(B_{k}\right) \backslash S} \Phi(u) \leq c_{k}-\varepsilon<c_{k}$, a contradiction.

\section{Applications}

As applications of our abstract theory, we consider the Dirichlet boundary value problem

$$
\left\{\begin{aligned}
-\Delta u & =f(x, u), & & \text { in } \Omega, \\
u & =0, & & \text { on } \partial \Omega,
\end{aligned}\right.
$$

where $\Omega \subset \mathbf{R}^{N}$ is a smooth bounded domain. We mainly want to demonstrate the types of results we may obtain by using our theory; so we shall not seek for optimal conditions placed on $f(x, u)$, although we may mention necessary changes in the proofs which provide more general results. Sign-changing solutions have attracted much attention in recent years; see [8], 9], 10, 12], [13, [14, 18, [19], 24, [25], [26], [27], 28], [40], [45], for various types of problems and [20], 29], 41] for numerical methods for sign-changing solutions.

We make the following assumption:

$\left(f_{1}\right) . f \in C^{1}(\bar{\Omega} \times \mathbf{R})$ and there are $C>0$ and $2 \leq \alpha<2^{*}=\frac{2 N}{N-2}$ (for $N=1,2$, we take $2^{*}=\infty$ ) such that

$$
\left|\frac{\partial f}{\partial u}(x, u)\right| \leq C\left(1+|u|^{\alpha-2}\right), \forall(x, u) \in \Omega \times \mathbf{R} .
$$

Under $\left(f_{1}\right)$ classical solutions of (4.1) are in one-to-one correspondence with critical points of the following $C^{2}$-functional on $E=H_{0}^{1}(\Omega)$ :

$$
\Phi(u)=\frac{1}{2} \int_{\Omega}|\nabla u|^{2} d x-\int_{\Omega} F(x, u) d x, u \in E,
$$

where $F(x, u)=\int_{0}^{u} f(x, \tau) d \tau$.

On $E$, let us define

$$
P_{E}=\{u \in E \mid u(x) \geq 0, \text { a.e. in } \Omega\},
$$


which is a closed convex cone. Let $X=C_{0}^{1}(\Omega)$. Then $X$ is a Banach space and embedded densely in $E$. Let

$$
P=P_{E} \cap X
$$

Then $P$ is a closed convex cone in $X$. Furthermore, $P=\stackrel{\circ}{P} \cup \partial P$ under the topology of $X$, i.e., there exist interior points in $P$. As we did in Section 3, we may define a partial order relation: $u, v \in X, u>v \Longleftrightarrow u-v \in P \backslash\{0\} ; u \gg v \Longleftrightarrow u-v \in \stackrel{\circ}{P}$.

Proposition 4.1. Under $\left(f_{1}\right),(\Phi)$ is satisfied.

Proof. By the elliptic theory, $K(\Phi) \subset C_{0}^{2, \alpha}(\Omega) \subset X$. A direct computation gives

$$
\nabla \Phi(u)=u-(-\Delta)^{-1} f(x, u):=u-G_{E}(u),
$$

where $G_{E}: E \rightarrow E$ is $C^{1}$ and compact. $G_{E}(X) \subset X$ and $G=\left.G_{E}\right|_{X}: X \rightarrow X$ is of class $C^{1}$, and $G$ is strongly order preserving in the sense that $u>v \Rightarrow G(u) \gg$ $G(v)$.

$\left(f_{2}\right) . \exists C>0$ such that

$$
\frac{f(x, t)-f(x, s)}{t-s} \geq-C, \forall x \in \Omega, t>s .
$$

Proposition 4.2. Under $\left(f_{1}\right)$ and $\left(f_{2}\right)$, if $\Phi$ satisfies the (PS) condition on $E$, then $W=P$ is an admissible invariant set for $\Phi$.

Proof. Using the constant $C$ from $\left(f_{2}\right)$, we may rewrite (4.1) as

$$
\begin{cases}-\Delta u+(C+1) u=(C+1) u+f(x, u), & \text { in } \Omega, \\ u=0, & \text { on } \partial \Omega\end{cases}
$$

Using the equivalent norm $\|u\|^{2}=\int_{\Omega}|\nabla u|^{2}+(C+1) u^{2}$, we see that

$$
\nabla \Phi(u)=u-\widetilde{G}_{E}(u),
$$

where

$$
\tilde{G}_{E}(u)=(-\Delta+C+1)^{-1}((C+1) u+f(x, u))=(-\Delta+C+1)^{-1} \widetilde{f}(x, u),
$$

in which $\tilde{f}(x, u)=(C+1) u+f(x, u)$ and thus $\tilde{f}(x, t)-\tilde{f}(x, s) \geq(t-s)$ for $t \geq s$. By the strong maximum principle, the restriction $\widetilde{G}$ of $\widetilde{G}_{E}$ on $X$, is strongly order preserving. Thus $P$ and $-P$ are invariant sets of the negative gradient flow of $\Phi$. The requirement (a) is satisfied automatically. For (d), we note that for all $v \in P \backslash\{0\}$, the vector field $-\nabla \Phi$ points at $v$ inside the cone $\stackrel{\circ}{P}$, and we have

$$
v-\nabla \Phi(v) \in \stackrel{\circ}{P}
$$

which follows from $v-\nabla \Phi(v)=\widetilde{G}(v) \gg 0$. To prove (b), let $u_{n}=\sigma\left(t_{n}, v\right)$ for some $v \in X \backslash(P \cup(-P))$ and let $t_{n} \rightarrow \infty$ be a sequence such that $u_{n} \rightarrow u$ in $E$ for some $u \in K(\Phi)$. Let $\xi(u)=\frac{1}{1+\|\nabla \Phi(u)\|^{2}}$ in $(2.1)$. Note that

$$
\sigma(t, v)=e^{-\theta(t, v)} v+e^{-\theta(t, v)} \int_{0}^{t} e^{\theta(s, v)} \widetilde{G}(\sigma(s, v)) \xi(\sigma(s, v)) d s,
$$

where $\theta(t, v)=\int_{0}^{t} \xi(\sigma(s, v)) d s$.

Let $X_{0}=W^{2, q}(\Omega)$ for some $q \geq 2$ such that the embedding from $X_{0}$ into $X=C_{0}^{1}(\Omega)$ is compact. 
It is well known that there exists a finite sequence of Banach spaces $X_{1}, \ldots, X_{m}$ such that

$$
X_{0} \hookrightarrow X_{1} \hookrightarrow \cdots \hookrightarrow X_{m}=E,
$$

the embeddings from $X_{k-1}$ into $X_{k}$ are continuous, $\widetilde{G}\left(X_{k}\right) \subset X_{k-1}$, and $\widetilde{G} \in$ $C\left(X_{k}, X_{k-1}\right)$ maps bounded sets to bounded sets. This is related to the notion of $X$-regularity as used in [33] and [15], and similar ideas were used also in [8], [9], [10], [16], [26].

By the (PS) condition,

$$
\sup _{t \geq 0}\|\sigma(t, v)\|_{E}=B_{m}<\infty .
$$

We need to show that the set

$$
\left\{e^{-\theta(t, v)} \int_{0}^{t} e^{\theta(s, v)} \widetilde{G}(\sigma(s, v)) \xi(\sigma(s, v)) d s \mid t \geq 0\right\}
$$

is compact in $X$. It suffices to show this set is bounded in $X_{0}$, since the embedding from $X_{0}$ into $X$ is compact. This is done by induction. First,

$$
\begin{aligned}
& \left\|e^{-\theta(t, v)} \int_{0}^{t} e^{\theta(s, v)} \widetilde{G}(\sigma(s, v)) \xi(\sigma(s, v)) d s\right\|_{X_{m}-1} \\
& \quad \leq e^{-\theta(t, v)} \int_{0}^{t} e^{\theta(s, v)} \xi(\sigma(s, v))\|\widetilde{G}(\sigma(s, v))\|_{X_{m-1}} d s \\
& \quad \leq B_{m-1},
\end{aligned}
$$

for some $B_{m-1}>0$. By induction, we have

$$
\left\|e^{-\theta(t, v)} \int_{0}^{t} e^{\theta(s, v)} \widetilde{G}(\sigma(s, v)) \xi(\sigma(s, v)) d s\right\|_{X_{0}} \leq B_{0} .
$$

The proof of (b) is complete. For (c), if $u_{n} \in K(\Phi) \cap(P \cup(-P))$ and $u_{n} \rightarrow u$ in $E$, then $u_{n}$ is bounded in $E$. By condition $\left(f_{1}\right)$, the elliptic theory, and the bootstrap argument, we get that $u_{n}$ is bounded in $W^{2, q}(\Omega)$ for some $q$ large such that the embedding from $W^{2, q}(\Omega)$ into $X$ is compact and thus $u_{n} \rightarrow u$ in $X$.

Let $\lambda_{1}<\lambda_{2}<\lambda_{3}<\cdots$ be the eigenvalues of $(-\Delta)$ in $\Omega$ with the Dirichlet condition, and denote this set by $\sigma(-\Delta)$. We make more assumptions:

$\left(f_{3}\right) . \varlimsup_{|t| \rightarrow \infty} \frac{f(x, t)}{t}<\lambda_{1}$ uniformly in $x \in \Omega$;

$\left(f_{4}\right) . \underline{\lim }_{|t| \rightarrow 0} \frac{f(x, t)}{t}>\lambda_{k}$ uniformly in $x \in \Omega$ for some $k \geq 2$;

$\left(f_{5}\right) . f(x, u)$ is odd in $u$.

Theorem 4.1 (Sublinear nonlinearity). Under $\left(f_{1,2,3,4}\right)$, equation (4.1) has at least one pair of one-sign solutions $u_{1}>0,-u_{1}<0$, and at least $(n-1)$ pairs of sign-changing solutions $\pm u_{i}$, for $i=2, \ldots, n$. Here $n=\sum_{i=1}^{k} \operatorname{dim} \operatorname{Ker}\left(-\Delta-\lambda_{i}\right)$.

Proof. It is easy to see that $\Phi \in C^{2}$, and by Proposition 4.1 ( $\Phi$ ) holds. Also $\Phi$ is even, $\Phi(0)=0$, bounded from below by $\left(f_{3}\right)$. $P$ is an admissible invariant set for $\Phi$. $\left(f_{4}\right)$ implies that when we take $F=\bigoplus_{i=1}^{k} \operatorname{Ker}\left(-\Delta-\lambda_{i}\right)$, we get $\sup _{F \cap \partial B_{\rho}} \Phi(x)<0$ for $0<\rho$ small. By Theorem 2.1, the result follows.

Remark 4.1. Equation (4.1) was studied for example in [18], [33], [44, and recently for sign-changing solutions in [17], [25]. Condition $\left(f_{2}\right)$ may be removed using a $C^{1}$ modification of $f$ which satisfies $\left(f_{2}\right)$ (see 33] for details). In [27] two pairs of sign-changing solutions are obtained under conditions similar to those of Theorem 4.1 . 
Next we consider a Landesman-Lazer type result.

$\left(f_{6}\right) . f(x, u)=\hat{\lambda} u+p(x, u)$ with $\hat{\lambda} \in \sigma(-\Delta)$.

$\left(f_{7}\right)$. There is $M>0$ such that

$$
|f(x, u)-\hat{\lambda} u| \leq M, \forall(x, u) \in \Omega \times R .
$$

$\left(f_{8}\right) . \int_{\Omega}\left(F(x, \phi)-\frac{\hat{\lambda}}{2} \phi^{2}\right) d x \rightarrow-\infty$ as $\|\phi\| \rightarrow \infty$ for $\phi \in \operatorname{Ker}(-\Delta-\hat{\lambda})$.

$\left(f_{9}\right)$. There is $r>0$ such that

$$
F(x, u)-\frac{\hat{\lambda}}{2} u^{2}>0, \forall(x, u) \in \Omega \times(0, r] .
$$

Theorem 4.2 (Asymptotically linear case with resonance). Under $\left(f_{1,2,5,6,7,8,9}\right)$, equation (4.1) has at least $n$ pairs of sign-changing solutions provided $\hat{\lambda} \neq \lambda_{1}$, where $n=\operatorname{dim} \operatorname{Ker}(-\Delta-\hat{\lambda})$.

Proof. For this problem, we use Theorem 2.3. Let $\hat{\lambda}=\lambda_{k}$ for some $k>1$. Let $F=\bigoplus_{i=1}^{k} \operatorname{Ker}\left(-\Delta-\lambda_{i}\right), H=\bigoplus_{i=k}^{\infty} \operatorname{Ker}\left(-\Delta-\lambda_{i}\right)$. Then $\operatorname{dim} F-\operatorname{codim} H=$ $\operatorname{dim} \operatorname{Ker}\left(-\Delta-\lambda_{k}\right)$. We can show similarly that $\sup _{F \cap \partial B_{\rho}} \Phi(x)<0$ for $\rho>0$ small, and by $\left(f_{7-8}\right), \inf _{H} \Phi(x)>-\infty$. Then the result follows from Theorem 2.3.

Remark 4.2. There are many papers in the literature related to the LandesmanLazer type problems. We just refer to [18] and 44] for references.

Next, we consider an asymptotically linear problem.

$\left(f_{10}\right) . \lim _{|t| \rightarrow \infty} f^{\prime}(x, t) \in\left[\lambda_{k}+\varepsilon, \lambda_{k+1}-\varepsilon\right]$, for some $\varepsilon>0$.

$\left(f_{11}\right) . \lim _{|t| \rightarrow 0} f^{\prime}(x, t) \in\left[\lambda_{l}+\varepsilon, \lambda_{l+1}-\varepsilon\right]$ for some $\varepsilon>0$.

Theorem 4.3 (Asymptotically linear case without resonance). Under conditions $\left(f_{1,2,5,10,11}\right)$, equation (4.1) has at least $n((n-1)$, resp.) pairs of sign-changing solutions provided $\lambda_{k}>\lambda_{l+1}$ or $\lambda_{l}>\lambda_{k+1}$ with $\min \{k, l\} \geq 1(\min \{k, l\}=0$, resp.). Here

$$
n=\sum_{i=l+1}^{k} \operatorname{dim} \operatorname{Ker}\left(-\Delta-\lambda_{i}\right), \quad \text { if } \lambda_{k}>\lambda_{l+1}
$$

and

$$
n=\sum_{i=k+1}^{l} \operatorname{dim} \operatorname{Ker}\left(-\Delta-\lambda_{i}\right), \quad \text { if } \lambda_{l}>\lambda_{k+1} .
$$

Using Theorem 2.2 or 2.3 , the proof is similar to the proof of Theorem 4.2 , and we omit it here.

Remark 4.3. Without the condition of $f$ being odd, for sign-changing solutions this problem has been studied for example in [10], [9], [13], [27], [39]; see the references therein too.

Next, we consider a nonlinear eigenvalue problem: for $r>0$ fixed,

$$
\begin{cases}-\Delta u=\lambda f(x, u), & \text { in } \Omega, \\ u=0 & \text { on } \partial \Omega, \\ \int_{\Omega}|\nabla u|^{2} d x=r^{2} . & \end{cases}
$$

We want to find solutions of the form $(\lambda, u) \in \mathbf{R} \times X$. 
$\left(f_{12}\right) . f(x, t) t>0$ for $(x, t) \in \Omega \times \mathbf{R}$ if $t \neq 0$.

Theorem 4.4 (Nonlinear eigenvalue problem). Under $\left(f_{1,2,5,12}\right)$, (4.2) has infinitely many sign-changing solutions.

Proof. We follow the original idea for the setting up of the problem in [44]. Consider

$$
\Phi(u)=-\int_{\Omega} F(x, u) d x, u \in E
$$

then $\Phi \in C^{2}(E, R)$ and is even. Let $r>0$ be fixed. At a critical point $u$ of $\left.\Phi\right|_{\partial B_{r}}$, where $B_{r}=\{u \in E \mid\|u\| \leq r\}$, we have

$$
\Phi^{\prime}(u) v-\mu(u, v)=0=-\int_{\Omega} f(x, u) v d x-\mu \int_{\Omega} \nabla u \cdot \nabla v d x
$$

for all $v \in E$. Choosing $v=u$ and using $\left(f_{12}\right)$, we get

$$
\mu=\frac{\Phi^{\prime}(u) u}{r^{2}}=\frac{-\int_{\Omega} f(x, u) u d x}{r^{2}}<0 .
$$

Therefore $u$ is a solution of (4.2) with $\lambda=-\mu^{-1}$. This leads us to use Theorem 2.4 for $\left.\Phi\right|_{\partial B_{r}}$ to get solutions of (4.2). It is proved in 44] that $\left.\Phi\right|_{\partial B_{r}}$ satisfies $(P S)_{c}$ for all $c<0$. By the maximum principle, $K\left(\left.\Phi\right|_{\partial B_{r}}\right) \cap \partial P=\emptyset$ for $r>0$. It is left to show that $P \cap \partial B_{r}$ is an admissible invariant set for $\left.\Phi\right|_{\partial B_{r}}$. Since $P$ is a closed convex cone, (a) of definition 2.2 is satisfied. Let $\widetilde{\Phi}=\left.\Phi\right|_{\partial B_{r}}$. Then

$$
\nabla \widetilde{\Phi}(u)=\nabla \Phi(u)-\frac{\langle\nabla \Phi(u), u\rangle u}{\|u\|^{2}}
$$

Note that $-\nabla \Phi(u)=(-\Delta)^{-1} f(x, u):=G_{E}(u): E \stackrel{C^{1}}{\rightarrow} E$, and $G:=\left.G_{E}\right|_{X}: X \stackrel{C^{1}}{\rightarrow} X$ is strongly order preserving. For $u \in P \cap \partial B_{r}$ we have

$$
u-s \nabla \widetilde{\Phi}(u)=u\left(1+\frac{s\langle\nabla \Phi(u), u\rangle}{r^{2}}\right)+s G(u) \gg u
$$

for $s>0$ small. This gives that the flow given by $-\nabla \widetilde{\Phi}(u)$ on $X \cap \partial B_{r}$ leaves $P$ invariant, and in fact property (d) is satisfied.

To prove (b) we use the notation in the proof of Proposition 4.2 and consider (2.1): $\sigma^{\prime}(t, v)=-\xi(\sigma(t, v)) \nabla \tilde{\Phi}(\sigma(t, v))$. We note that we only need to consider a compact orbit (in $E$ ) of the negative gradient flow in $\tilde{\Phi}^{c}$ for some $c<0$, because all critical values we obtain will be negative ones. Define

$$
a(u):=-\frac{\langle\nabla \Phi(u), u\rangle}{r^{2}}=\int_{\Omega} f(x, u) u d x
$$

Then if $\sigma(t, v) \subset \tilde{\Phi}^{c}$ for some $c<0$, using the compact embedding from $E$ into $L^{\alpha}(\Omega)$ for $\alpha<2^{*}$, we get $a(\sigma(t, v)) \geq a_{0}>0$ for all $t \geq 0$. Since $-\nabla \tilde{\Phi}(u)=$ $-a(u) u+G(u)$, we get $\sigma^{\prime}(t, v)=-\xi(\sigma(t, v)) a(\sigma(t, v)) \sigma(t, v)+\xi(\sigma(t, v)) G(\sigma(t, v))$. Then we may follow essentially the same argument as in the proof of Proposition 4.2 to show that the orbit is compact in $X$. (c) can be proved by an argument similar to the one in the proof of Proposition 4.2.

Remark 4.4. We refer the reader to the classical treatment of nonlinear eigenvalue problems in [35], 44], and recent work on sign-changing solutions in [37.

We consider a superlinear problem next. Assume

$\left(f_{13}\right)$. There is $\mu>2$ such that $\mu F(u) \leq f(u) u$ for $|u|$ large. 
Theorem 4.5 (Superlinear nonlinearity). Under $\left(f_{1,2,5,13}\right)$, (4.1) has infinitely many sign-changing solutions.

Proof. Let $E_{i}=\operatorname{ker}\left(-\Delta-\lambda_{i}\right), Y_{k}=\bigoplus_{i=1}^{k} E_{i}$, and $Z_{k}=\bigoplus_{i=k}^{\infty} E_{i}$. Then it is easy to check that for all $k$ with $\lambda_{k}>f_{u}(x, 0)$ there are $\rho_{k}>r_{k}>0$ such that $\sup _{Y_{k} \cap B_{\rho_{k}}(0)} \Phi(u) \leq a_{k}:=0$ and $b_{k}:=\inf _{Z_{k} \cap \partial B_{r_{k}}(0)} \Phi(u)>0$ and $b_{k} \rightarrow \infty$. Thus $Y_{k} \subset X$ for any $k \geq 1$. Moreover, for $k \geq 2, Z_{k} \cap P=\{0\}$. This can be seen by noting that for all $u \in P \backslash\{0\}, \int_{\Omega} u \phi_{1}(x) d x>0$, while for $u \in Z_{k}$, $\int_{\Omega} u \phi_{1}(x) d x=0$, where $\phi_{1}$ is the first eigenfunction of the Laplacian operator on $\Omega$. Applying Theorem 3.2, we get $K_{c_{k}} \backslash(P \cup(-P)) \neq \emptyset$ for all large $k$, where $c_{k}$ is defined in the proof of Theorem 3.2.

Remark 4.5. The existence of infinitely many sign-changing solutions for this problem was also given recently in [8, in which a different method was used. Our method here is more intuitive and constructive, along the line of the classical treatment in 6].

Next, we give an example in which the functional $\Phi$ cannot be $C^{2}$ and approximations by $C^{2}$ functionals can be done to use our theory to get multiple sign-changing solutions. The problem is

$$
\left\{\begin{aligned}
-\Delta u & =\lambda|u|^{q-2} u+f(u), & & \text { in } \Omega \\
u & =0, & & \text { on } \partial \Omega,
\end{aligned}\right.
$$

where $\lambda>0$ is a real number, $1<q<2$. Note that $\widetilde{f}(u)=\lambda|u|^{q-2} u+f(u) \in C^{q-1}$ in $u$. So $\Phi$ is in $C^{1}(E, \mathbf{R})$.

Then under $\left(f_{1}\right)$ and $\left(f_{2}\right)$, we have

$$
\nabla \Phi(u)=u-G_{E}(u),
$$

where $G_{E}(u)=(-\Delta)^{-1} \widetilde{f}(u): E \rightarrow E$ is only $C^{0}$, and $G:=\left.G_{E}\right|_{X}: X \rightarrow X$ is also only $C^{0} . G(X) \subset X$ follows because if $u \in C_{0}^{1}(\Omega)$, then $\widetilde{f}(x, u) \in C_{0}^{\alpha}(\Omega)$, where $\alpha=q-1$ and $G(u) \in C^{2, \alpha}(\Omega)$.

Theorem 4.6 (Concave nonlinearity near zero). Under $\left(f_{1,2,3,5}\right)$, for every $\lambda>0$, (4.3) has a pair of positive and negative solutions $u_{+}$and $u_{-}=-u_{+}$, and has infinitely many sign-changing solutions.

Proof. Let us fix $k$, an integer, and $\alpha+f^{\prime}(0)>\lambda_{k}$, the $k$-th engenvalue of $-\Delta$.

Consider $g_{h}(u)=\alpha u$ for $|u| \leq h$ and $g_{h}(u)=\lambda|u|^{q-2} u$ for $|u| \geq 2 h$, with a smooth connection for $h \leq|u| \leq 2 h$, so that $g_{h}(u) \rightarrow \lambda|u|^{q-2} u$ as $h \rightarrow 0$ in the continuous norm. We may assume $g_{h} \leq g_{h^{\prime}}$ if $h \geq h^{\prime}$. Then we can consider the corresponding equation with $\lambda|u|^{q-2} u+f(u)$ replaced by $g_{h}(u)+f(u)$, and the corresponding functional $\Phi_{h}(u)$ is in $C^{2}(E, \mathbf{R})$. It is easy to check that for all $u \in E$, $0 \leq \Phi_{h}(u)-\Phi(u) \leq C h^{q+1}$ and $\left\|\nabla \Phi_{h}(u)-\nabla \Phi(u)\right\| \leq C h^{q}$, where $C$ depends only on $\Omega$ and $\lambda$. Applying Theorem 2.1 to $\Phi_{h}$, we get $c_{2}(h) \leq c_{3}(h) \leq \ldots \leq c_{k}(h)<0$, which give $(k-1)$ pairs of sign-changing solutions for the modified equations.

For $\Phi$ we may also define $c_{2} \leq c_{3} \leq \ldots \leq c_{k}$ as for $c_{i}(h), i=2,3, \ldots, k$. Then it is not difficult to show for $i=2,3, \ldots, k$ we have $c_{i}(h) \geq c_{i}$ and $c_{i}(h) \rightarrow c_{i}$ as $h \rightarrow 0$. Using the fact that for $h$ small all critical points of $\Phi_{h}$ are uniformly bounded in $E$, we can show that $K_{c_{i}}(\Phi) \cap S \neq \emptyset$. In fact, let $u_{h} \in K_{c_{i}(h)}\left(\Phi_{h}\right)$ and $u_{h} \in S$. Using the elliptic theory, we get $u_{h} \rightarrow u$ in $E$ and in $X$ such that $u$ is a solution $(4.3)_{\lambda}$. Since there is no solution belonging to $\partial(P \cup(-P))$, we get $u \in S$ and $\Phi(u)=c_{i}$. 
Thus, if the $c_{i}$ 's are all different, we get at least $(k-1)$ sign-changing solutions for (4.3).

Let us assume $c:=c_{i+1}=\cdots=c_{i+l}$ for some $l>1$. Let $\delta>0$ be such that $\operatorname{dist}_{E}\left(K_{c}^{1}(\Phi), K_{c}^{2}(\Phi)\right) \geq 8 \delta>0, i_{E}\left(\overline{\left(K_{c}^{2}(\Phi)\right)_{4 \delta}}\right)=i_{E}\left(K_{c}^{2}(\Phi)\right)$ and for all $j=$ $i+1, \ldots, i+l$ and $h$ small, $\left(K_{c_{j}(h)}^{2}\left(\Phi_{h}\right)\right)_{3 \delta} \subset\left(K_{c}^{2}(\Phi)\right)_{4 \delta}$. By the convergence of $\Phi_{h}$ to $\Phi$, there is $\varepsilon_{0}>0$ such that we can make (2.2) and (2.3) hold uniformly for all $\Phi_{h}$ with $h$ small, and all $c_{j}(h)$ with $j=i+1, \ldots, i+l$. Note that $\varepsilon_{0}$ in Lemma 2.4 is determined by (2.2) and (2.3). Thus we may use Lemma 2.4 for all $\Phi_{h}$ with $h$ small and all $c_{j}(h)$ with $j=i+1, \ldots, i+l$. Take $A \in \Sigma_{i+l}$ such that $\sup _{A \cap S} \Phi(u) \leq c+\frac{\varepsilon}{2}$ with $\varepsilon=\frac{\varepsilon_{0}}{2}$. Then for $h$ small, $\sup _{A \cap S} \Phi_{h}(u) \leq c_{i+l}(h)+\varepsilon$. Then, applying Lemma 2.4 to $\Phi_{h}$, there is $\eta \in C([0,1] \times X, X)$ such that

$$
\eta\left(1, A \backslash\left(K_{c_{i+l}(h)}^{2}\left(\Phi_{h}\right)\right)_{3 \delta}\right) \subset \Phi_{h}^{c_{i+l}(h)-\varepsilon} \cup(P \cup(-P)) .
$$

We may assume $c_{i+l}(h)-\varepsilon \leq c-\frac{\varepsilon}{2}$, since as $h \rightarrow 0, c_{i+l}(h) \rightarrow c$. Then we have

$$
i_{X}\left(\eta\left(1, A \backslash\left(K_{c_{i+l}(h)}^{2}\left(\Phi_{h}\right)\right)_{3 \delta}\right)\right) \leq i,
$$

for if not we get $c_{i+1}(h) \leq c_{i+l}(h)-\varepsilon \leq c-\frac{\varepsilon}{2}<c=c_{i+1}$, which is a contradiction with $c_{i+1}(h) \geq c_{i+1}$. Finally, let $N=\overline{\left(K_{c_{i}(h)}^{2}\right)_{3 \delta}}$; we have

$$
\begin{aligned}
& i+l \leq i_{X}(A) \leq i_{X}(A \backslash(\stackrel{\circ}{N} \cap X))+i_{X}(N \cap X) \\
& \leq i_{X}\left(\eta\left(1, A \backslash\left(K_{c_{i}(h)}^{2}\right)_{3 \delta}\right)+i_{E}(N) \leq i+i_{E}\left(\overline{\left(K_{c}^{2}(\Phi)\right)_{4 \delta}}\right)=i+i_{E}\left(K_{c}^{2}\right),\right.
\end{aligned}
$$

which gives $i_{E}\left(K_{c} \cap S\right) \geq l$.

This proves that $\Phi$ has at least $(k-1)$ pairs of sign-changing solutions. Since $k$ is arbitrary, the proof is complete.

Remark 4.6. Using the method of [48], we may only need that $\left(f_{1}\right)$ is satisfied for $|t|$ small in a neighborhood of $t=0$. We leave the statement to interested readers. The radial case was considered in [5]. Results for this type of problems under global conditions on $f$ were given in [4], 6], [31, and for sign-changing solutions in [39].

Finally, we consider a problem with a parameter which may have more and more solutions as the parameter changes:

$$
\left\{\begin{aligned}
-\Delta u & =\lambda f(u), & & \text { in } \Omega, \\
u & =0, & & \text { on } \partial \Omega,
\end{aligned}\right.
$$

where $\lambda>0$ is a real number. We make one more assumption.

$\left(f_{14}\right) . f(u)=o(|u|)$ for $u$ small, and there is $r>0$ such that $f(u)>0$ for $u \in(0, r)$ and $f(r)=0$.

Theorem 4.7. Under $\left(f_{1,5,14}\right)$, for every integer $k$ there is $\bar{\lambda}_{k}>0$ such that for $\lambda>\bar{\lambda}_{k},(4.4)_{\lambda}$ has at least $2(k-1)$ pairs of sign-changing solutions, $(k-1)$ pairs of which have positive critical values and $(k-1)$ pairs of which have negative critical values. In addition, there is $\bar{\lambda}>0$ such that for all $\lambda>\bar{\lambda},(4.4)_{\lambda}$ has two positive (and two negative) solutions, with one having positive critical value and the other having negative critical value.

Proof. First we truncate $f$ to 0 for $|u| \geq r$. Then $f$ is Lipschitz continuous and the corresponding functional $\Phi$ is $C^{2-0}$. Note that $\left(f_{2}\right)$ is satisfied for the truncated $f$ by $\left(f_{1}\right)$ and $\left(f_{14}\right)$. We shall use Theorem 2.1 to get $(k-1)$ pairs of sign-changing solutions having negative critical values, and Theorem 2.3 to get $(k-1)$ pairs of 
sign-changing solutions having positive critical values. Let $k$ be fixed, and choose $\lambda_{m}$ such that $\operatorname{dim} F \geq k$ when we write $F=\bigoplus_{i=1}^{m} \operatorname{Ker}\left(-\Delta-\lambda_{i}\right)$. Take $\rho>0$

so small that $\int_{\Omega} F(u) d x>0$ for all $u \in \partial B_{\rho}(0) \cap F$. Then there is $\bar{\lambda}_{k}>0$ such that for all $\lambda>\bar{\lambda}_{k}$ we have $\sup _{\partial B_{\rho}(0) \cap F} \Phi(u)<0$. Thus by Theorem 2,1 we obtain $(k-1)$ pairs of sign-changing solutions having negative critical values. Since 0 is a local minimum of $\Phi$, by Theorem 2.2 we also obtain $(k-1)$ pairs of sign-changing solutions having positive critical values. The existence of two positive solutions follows from the usual arguments by cutting off the negative part of $f(u)$ and using minimization to get the positive solution having negative critical value and using the Mountain Pass Theorem to get the positive solution having positive critical value.

Remark 4.7. This problem was treated in [6] and [4] without information about sign-changing solutions.

\section{REFERENCES}

1. S. Alama, M. Del Pino, Solutions of elliptic equations with indefinite nonlinearities via Morse theory and linking, Ann. Inst. H. Poincaré Analyse Non Linéaire 13(1996) 95-115. MR 96m:35091

2. S. Alama, G. Tarantello, On semilinear elliptic equations with indefinite nonlinearities, Calculus of Variations and Partial Differential Equations 1 (1993) 439-475. MR 97a:35057

3. H. Amann, Fixed point equations and nonlinear eigenvalue problems in ordered Banach spaces, SIAM Review, 18(1976) 620-709. MR 54:3519

4. A. Ambrosetti, J. Garcia Azorero, I. Peral, Multiplicity results for some nonlinear elliptic equations, Jour. Funct. Anal. 137 (1996) 219-242. MR 97b:35059

5. A. Ambrosetti, J. Garcia Azorero, I. Peral, Quasilinear equations with a multiple bifurcation, Differential Integral Equations, 10 (1997), 37-50. MR 97i:35036

6. A. Ambrosetti, H. Brezis, G. Cerami, Combined effects of concave and convex nonlinearities in some elliptic problems, Jour. Funct. Anal. 122(1994) 519-543. MR 95g:35059

7. A. Ambrosetti, P. Rabinowitz, Dual variational methods in critical point theory and applications, Jour. Funct. Anal. 14(1973) 349-381. MR 51:6412

8. T. Bartsch, Critical point theory on partially ordered Hilbert spaces, J. Funct. Anal. 186 (2001), 117-152. CMP 1863294

9. T. Bartsch, K.C. Chang, Z.-Q. Wang, On the Morse indices of sign-changing solutions for nonlinear elliptic problems, Math. Zeit., 233 (2000), 655-677. MR 2001c:35079

10. T. Bartsch, Z.-Q. Wang, On the existence of sign changing solutions for semilinear Dirichlet problems, Topological Meth. Nonlinear Anal., 7(1996) 115-131. MR 97m:35076

11. H. Brezis, L. Nirenberg, Remarks on finding critical points, Comm. Pure Appl. Math. 64(1991) 939-963. MR 92i:58032

12. A. Castro, J. Cossio, J.M. Neuberger, A sign changing solution for a superlinear Dirichlet problem, Rocky Mount. J. Math., 27 (1997), 1041-1053. MR 99f:35056

13. A. Castro, J. Cossio, J.M. Neuberger, A minmax principle, index of the critical point, and existence of sign-changing solutions to elliptic BVPs, Electron. J. Diff. Equations (1998), No. 2, 18 pp. MR 98j:35060

14. A. Castro, M. Finan, Existence of many sign-changing nonradial solutions for semilinear elliptic problems on thin annuli, Topological Meth. Nonlinear Anal. 13(1999), 273-279. MR 2000j:35092

15. K.C. Chang, A variant mountain pass lemma, Sci. Sinica Ser. A 26 (1983), no. 12, 1241-1255. MR 85h:58037

16. K.C. Chang, Variational methods and sub- and supersolutions, Sci. Sinica Ser. A 26 (1983), no. 12, 1256-1265. MR 85h:58038

17. K.C. Chang, Infinite dimensional Morse theory and multiple solution problems, Birkhäuser Boston 1993. MR 94e:58023 
18. K.C. Chang, Morse theory in nonlinear analysis. Nonlinear functional analysis and applications to differential equations (Trieste, 1997), 60-101, World Sci. Publishing, 1998. MR 2000k:58015

19. G. Cerami, S. Solimini, M. Struwe, Some existence results for superlinear elliptic boundary value problems involving critical exponents, J. Funct. Anal., 69(1986), 289-306. MR 88b:35074

20. G. Chen, W. Ni, J. Zhou, Algorithms and visualization for solutions of nonlinear elliptic equations, Internat. Jour. Bifur. Chaos Appl. Sci. Engrg., 10 (2000), 1565-1612. CMP 2001:01

21. D.C Clark, A variant of the Ljusternik-Schnirelman theory, Ind. Univ. Math. J. 22(1972) 65-74. MR 45:5836

22. E.N. Dancer, On the indices of fixed points of mappings in cones and applications, J. Math. Anal. Appl., 91(1983), 131-151. MR 84d:58020

23. E.N. Dancer, Positivity of maps and applications, Topological Nonlinear Analysis, Progr. Nonlinear Differential Equations Appl., 15(1995) 303-340, Birkhäuser, Boston. MR 95m:47119

24. E.N. Dancer, Y. Du, On sign-changing solutions of certain semilinear elliptic problems, Appl. Anal. 56 (1995), no. 3-4, 193-206. MR 97m:35061

25. E.N. Dancer, Y. Du, Multiple solutions of some semilinear elliptic equations via the generalized Conley index, J. Math. Anal. Appl. 189 (1995), no. 3, 848-871. MR 96a:35059

26. E.N. Dancer, Y. Du, The generalized Conley index and multiple solutions of semilinear elliptic problems, Abstract Appl. Anal., 1(1996) 103-135. MR 97i:35048

27. E.N. Dancer, Y. Du, A note on multiple solutions of some semilinear elliptic problem, J. Math. Anal. Appl. 211(1997) 626-640. MR 98g:35075

28. E.N. Dancer, S. Yan, On the profile of the changing sign mountain pass solutions for an elliptic problem, preprint.

29. Z. Ding, D. Costa, G. Chen, A high-linking algorithm for sign-changing solutions of semilinear elliptic equations, Nonlinear Analysis, 38 (1999), 151-172. MR 2000d:65208

30. E. Fadell, P.H. Rabinowitz, Generalized cohomological index thoeries for Lie group actions with an application to bifurcation questions for Hamiltonian systems, Inv. Math., 45 (1978), 139-174. MR 57:17677

31. J. Garcia Azorero, I. Peral Alonso, Multiplicity of solutions for elliptic problems with critical exponent or with a nonsymmetric term, Trans. AMS. 323 (1991), 877 - 895. MR 91g:35108

32. H. P. Heinz, Free Ljusternik-Schnirelman theory and the bifurcation diagrams of certain singular nonlinear systems, J. Diff. Eqns. 263 - 300 (1987). MR 88d:34020

33. H. Hofer, Variational and topological methods in partially ordered Hilbert spaces, Math. Ann. 261 (1982), 493 - 514. MR 84g:58030

34. H. Hofer, A note on the topological degree at a critical point of mountain pass type, Proc. AMS 90(1984) 309-315. MR 85a:58015

35. M.A. Krasnoselskii, Topological Methods in the Theory of Nonlinear Integral Equations, Pergamon, Oxford, New York (1964). MR 28:2414

36. S.J. Li, Some aspects of semilinear elliptic boundary value problem, Progress in Nonlinear Analysis, (1999) 234-256.

37. Y. Li, Z.L. Liu, Multiple and sign-changing solutions of an elliptic eigenvalue problem with constraint, Science in China (Series A), 44(2001), No.1, 48-57. MR 2002b:35166

38. S.J. Li, Z.-Q. Wang,. An abstract critical point theorem and applications, Acta Math. Sinica, 29(1986), 585-589. (Chinese) MR 88a:58036

39. S.J. Li, Z.-Q. Wang, Mountain-pass theorem in order intervals and multiple solutions for semilinear elliptic Dirichlet problems, J. d'Analyse Math. 81(2000) 373-396. MR 2001h:35063

40. Y. Li and J. Zhou, A minimax method for finding multiple critical points and its applications to semilinear PDEs, SIAM J. Sci. Comput. 23 (2001), no. 3, 840-865.

41. J. M. Neuberger, A numerical method for finding sign-changing solutions of superlinear Dirichlet problems, Nonlinear World 4 (1997), 73-83. MR 98c:65200

42. R.S. Palais, Lusternik-Schnirelman theory on Banach manifolds, Topology, 5(1966), 1-16. MR 45:4184

43. P. Rabinowitz, Some critical point theorems and applications to semilinear elliptic partical differential equations, Ann. Scuola Norm. Sup. Pisa, Seri IV. 5 (1978) 215-223. MR 58:7695

44. P. Rabinowitz, Minimax Methods in Critical Point Theory with Applications to differential Equations, CBMS Reg. Conf. Ser. Math. 65, American Mathematical Society, Providence, R.I., (1986). MR 87j:58024 
45. G. Tarantello, Nodal solutions of semilinear elliptic equations with critical exponent, Differential Integral Equations 5(1992), 25-42. MR 92k:35109

46. Z.-Q. Wang, A $Z_{p}$-index theory, Acta Math. Sinica (New Ser.), 6(1990), 18-23. MR 92a:58040

47. Z.-Q. Wang, On a superlinear elliptic equation, Ann. Inst. H. Poincaré Analyse Non Linéaire, 8(1991), 43 - 57. MR 92a:35064

48. Z.-Q. Wang, Nonlinear boundary value problems with concave nonlinearities near the origin, NoDEA Nonlinear Diff. Equations Appl. 8(2001), 15-33. MR 2002c:35107

49. Z.-Q. Wang, Sign-Changing Solutions for a Class of Nonlinear Elliptic Problems, Nonlinear Analysis(Eds. K.-C. Chang and Y. Long), Nankai Series in Pure and Applied Math. 6 (2000), 370-383.

50. M. Willem, Minimax Theorems, Birkhäuser (1996). MR 97h:58037

Institute of Mathematics, Academy of Mathematics and Systems Sciences, Academia SinicA, BeiJing 100080, P.R. China

E-mail address: lisj@math03.math.ac.cn

Department of Mathematics and Statistics, Utah State University, Logan, Utah 84322

E-mail address: wang@math.usu.edu 